\title{
Stem cell hierarchy in dorsal aorta
}

\author{
Bing Liu ${ }^{1}$, Hui-Yu Yao ${ }^{1}$, Yu Lan ${ }^{1}$, Xiao-Yan Wang ${ }^{1}$, Xiao Yang $^{1}$, Ning Mao ${ }^{1}$ \\ ${ }^{1}$ Institute of Basic Medical Sciences, Beijing, China* Institute of Biotechnology, Beijing, China
}

During embryogenesis, the aorta-gonad-mesonephros (AGM) region serves as an important niche for various kinds of stem cells, including hematopoietic stem cells (HSCs), primordial germ cells (PGCs), meso-angioblasts and angioblasts. Here, we report additional two types of stem cells that are in close association with definitive hematopoiesis in the AGM. While the hemogenic endothelium is well known as the precursor of AGM hematopoiesis, the hemangioblast, a cell type bearing hematopoietic and endothelial potential, is hypothesized for long time. To address the issue, we developed a unique three-step assay of high proliferative potential (HPP) precursors. The dorsal aorta plus its surrounding mesenchyme (AoM) other than the gonad and mesonephros (GM) contained clonal HPP precursors (CD45+ CD31-) with hemangioblastic characteristics. They displayed hematopoietic self-renewal ability and were readily to differentiate into functional endothelial cells in vitro, i.e. incorporating Ac-LDL and forming network structures in Matrigel. The clonal nature was verified by cell mixing assay. Surprisingly, the bipotential precursor, designated here as the HPP-HA, was not readily detected in the yolk sac (E8.25-E12.5), embryonic circulation (E10.5), placenta (E10.5-E11.5), fetal liver (E11.5-E12.5) and even umbilical artery (E11.5), reflective of its strictly spatial-regulated ontogeny. Intriguingly, the HPP-HA was detected in the umbilical artery when cocultured with AGM cells, suggesting the unique microenvironment cues in the AGM.

Mesenchymal stem cells (MSCs) are multi-potent stem cells that can generate various microenvironment components in bone marrow, ensuring a tight and precise control over self-renewal and multi-lineage differentiation of HSCs. Nevertheless, their spatiotemporal correlation with embryonic hematopoiesis remains unclear in mammalian embryos. We find that human AoM resided with bona fide MSCs. They were highly self-renewal as fibroblastoid population bearing uniform surface markers (CD45-, CD34-, SH2+, SH3+, CD29+, and CD44+), expressed pluri-potential molecules Oct-4 and Nanog, and clonally demonstrated tri-lineage differentiation capacity (osteocytes, chondrocytes and adipocytes). Moreover, they were functionally equivalent to MSCs from adult bone marrow, i.e., maintaining long-term hematopoiesis and suppressing $\mathrm{T}$ lymphocyte proliferation in vitro. In comparison, the yolk sac contained bi-potent mesenchymal precursors that propagated more slowly and failed to generate chondrocytes in vitro. The finding was in striking contrast to the mouse yolk sac showing neither adipogenic nor osteogenic potential. Together with previous knowledge, we propose that typical MSCs, like adult-type HSCs, initially develop in the AGM prior to their emergence in fetal liver and marrow.

To understand the regulatory mechanisms of endothelial niche on the development of hemangioblast as well as MSC, we focused on the transforming growth factor- $\beta$ (TGF- $\beta$ )/Smad signaling pathway. Here, we deleted Smad4 gene, a central mediator of TGF- $\beta$ signaling, specifically in endothelial cells (ECs) using the Cre-LoxP system. ECspecific Smad4 mutant mice died at embryonic day 10.5 due to cardiovascular defects including attenuated vessels sprouting and remodeling, and collapsed dorsal aorta. Noticeably, Smad4-deficient ECs demonstrated an intrinsic defect in tube formation in vitro. Furthermore, Smad4-deficient ECs dissociated away from the surrounding cells and the mutant embryos suffered from impaired development of vascular smooth muscle cells. The disturbed vascular integrity and maturation in Smad4 mutant mice was associated with altered expression of angiopoietins and downregulation of a gap junction component, connexin43. Our ongoing work is investigating whether the development of hemangioblast and MSC are defective in the mutant embryos.

Cell Research (2008) 18:s54. doi: 10.1038/cr.2008.144; published online 4 August 2008

Correspondence: Bing Liu

Tel: 010-66931320, 13621068177

E-mail: bingliu17@yahoo.com 